\title{
Muséologies
}

Les cahiers d'études supérieures

\section{Jacques Des Rochers, conservateur de l'art canadien, Musée des beaux-arts de Montréal}

\section{Catherine Fortin et Alessandra Mariani}

Volume 4, numéro 1, automne 2009

URI : https://id.erudit.org/iderudit/1033533ar

DOI : https://doi.org/10.7202/1033533ar

Aller au sommaire du numéro

Éditeur(s)

Association Québécoise de Promotion des Recherches Étudiantes en

Muséologie (AQPREM)

ISSN

1718-5181 (imprimé)

1929-7815 (numérique)

Découvrir la revue

Citer ce document

Fortin, C. \& Mariani, A. (2009). Jacques Des Rochers, conservateur de l'art canadien, Musée des beaux-arts de Montréal. Muséologies, 4(1), 78-91.

https://doi.org/10.7202/1033533ar d'utilisation que vous pouvez consulter en ligne. 
Dialogue deux

Jacques Des Rochers,

conservateur de l'art canadien,

Musée des beaux-arts de Montréal

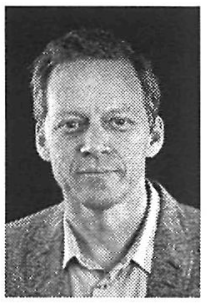

[Réalisé par Catherine Fortin et Alessandra Mariani le 6 novembre 2009] 
CATHERINE ForTIN \& ALESSANDRA MARIANI Pouvez-vous décrire votre parcours professionnel jusqu'à vos fonctions actuelles?

\section{Jacques Des Rochers}

Il s'agit d'un parcours à entrées multiples qui a fait de moi une personne au profil relativement hybride. Je constate aujourd'hui que la colonne vertebrale de ce parcours est un intérêt indéniable pourles questions de patrimoine identitaire, de l'artefact issu de contextes archéologiques, et de l'archive à l'espace urbain intégrant bien entendu, l'œuvre d'art. J'ai complété ma formation à l'Université Laval et à l'Université de Montréal, en archéologie et études anciennes, en histoire de l'art, en arts visuels et en patrimoine et aménagement urbain. Au cours des années 1980,j'étais principalement intéressé par la pratique des arts, tout particulièrement avec la photographie et l'histoire comme media.

Comme grand nombre d'artistes à l'époque, je m'intéressais aux questions liées à la fabrication de l'œuvre. Tous les aspects de la manipulation et de la construction matérielle étaient considérés comme un apport au contenu de celle-ci. Il s'agissait aussi d'un moment important dans le développement de la pratique de l'installation, de la mise en espace des œuvres, qu'elles soient ou non in situ. Ma réflexion personnelle s'orientait alors vers les questions de mémoire et d' "aléatoire", de palimpseste et d'accumulation et celles de la collection et du sacré dans l'art. J'ai exposé pendant une dizaine d'années, obtenu des bourses, un studio à l'étranger où j'ai développé un intérêt accru pour le paysage et l'aménagement du territoire...; les études que j'ai poursuivies par la suite dans ce domaine m'ont permis de développer une expertise diversifiée dans l'évaluation de la préservation et de la mise en valeur du patrimoine urbain. La Ville de Montréal m'a approché pour travailler à l'élaboration d'outils de référence en vue d'une première politique municipale du patrimoine montréalais. La publication en 1998 de l'ouvrage Le patrimoine de Montréal. Document de référẹnce est issue de cette expérience.J'ai ensuite œuvréa mon compte à la réalisation d'un nombre significatifd'études et d'analyses patrimoniales pour divers organismes publics et privés tels que la Commission des biens culturels, le ministère de la Culture et des Communications du Québec, la Ville de Montréal, des agences d'urbanisme et d'architecture et des communautés religieuses. Dans ce contexte, j'ai collaboré à plusieurs publications spécialisées sur l'ensemble $d u$ patrimoine montréalais.

C'est grâce à mon implication dans la préservation et la mise en valeur du patrimoine religieux que j'ai été embauché en juin 2002 en tant que conservateur de l'art canadien et religieux par le Musée des beaux-arts de Montréal, alors qu'avait été considérée l'idée d'ouvrir un pavillon dédié à l'art religieux canadien dans l'église Erskine and American. 
CATHERINE ForTIN \& AlessandRA MARIANI En quoi consiste votre travail comme conservateur de l'art canadien?

JACQUES Des Rochers

Mes tâches sont fascinantes et diversifiées. Je touche à toutes les étapes de l'enrichissement de la collection et de sa mise en valeur, dans un cadre comparatif des plus précieux. En fait, comme la collection du musée est de nature encyclopédique, toutes acquisitions, expositions ou publications bénéficient de la synergie de projets et d'activités des autres collègues à l'interne et de leur indispensable expertise, qu'elles soient de nature archivistique, documentaire, éditoriale, éducationnelle, communicationnelle ou qu'elles soient liées aux services de restauration ou de design. Il est aussi essentiel de construire et de maintenir de bons contacts avec les collectionneurs, dans le but de poursuivre l'enrichissement de la collection. Pour chaque acquisition considérée, qu'il s'agisse d'un don ou d'un achat, le conservateur doit effectuer une recherche documentaire (rapport d'acquisition) qui est par la suite déposée aux archives. J'ai ainsi produit, depuis 2002, quelques centaines de rapports. Les acquisitions majeures sont l'objet de textes que nous rédigeons pour les trois exemplaires annuels de la revue $M$, publication du musée qui est distribuée aux membres. Lors de la vingtaine de réunions annuelles du comité d'acquisition (composé de comités internes, avec la directrice et les conservateurs, et de comités externes, auxquels s'ajoutent des pairs et des proches du musée), les échanges sont déterminants. L'étendue des champs de collection de chaque conservateur au Musée des beaux-arts de Montréal favorise le travail avec des commissaires invités: c'est avec eux que j'ai coordonné des expositions sur des artistes comme James Wilson Morrice, Fritz Brandtner ou Sam Borenstein, de même que sur le galeriste et collectionneur montréalais Max Stern, produites au musée et mises en circulation par la suite, ou produites hors les murs - telle l'exposition Emily Carr - et exposées au musée. J'ai par ailleurs étéle commissaire de l'exposition L'Héritage artistique des Sulpiciens de Montréal, qui marquait le $350^{\circ}$ anniversaire de leur arrivée au pays. Cette exposition présentée dans l'église Erskine and American, ouverte pour la première fois au public par le musée, impliquait une appréciation des lieux tout autant que des collections, en isolant le regard surces deux objets aux proportions et aux attributs différents. Nous voulions alors qu'il y ait une démarcation nette entre le design contemporain du projet et le décor Arts and Crafts de l'église. C'est le défi considérable $q u$ 'ont su relever l'architecte Pierre Thibault et les équipes du musée. La nouvelle mise en valeur de la collection d'art inuit avec le concours de l'architecte Jacques Rousseau nous a aussi permis une autre lecture de ce riche corpus du musée, tout comme la présentation des œuvres 


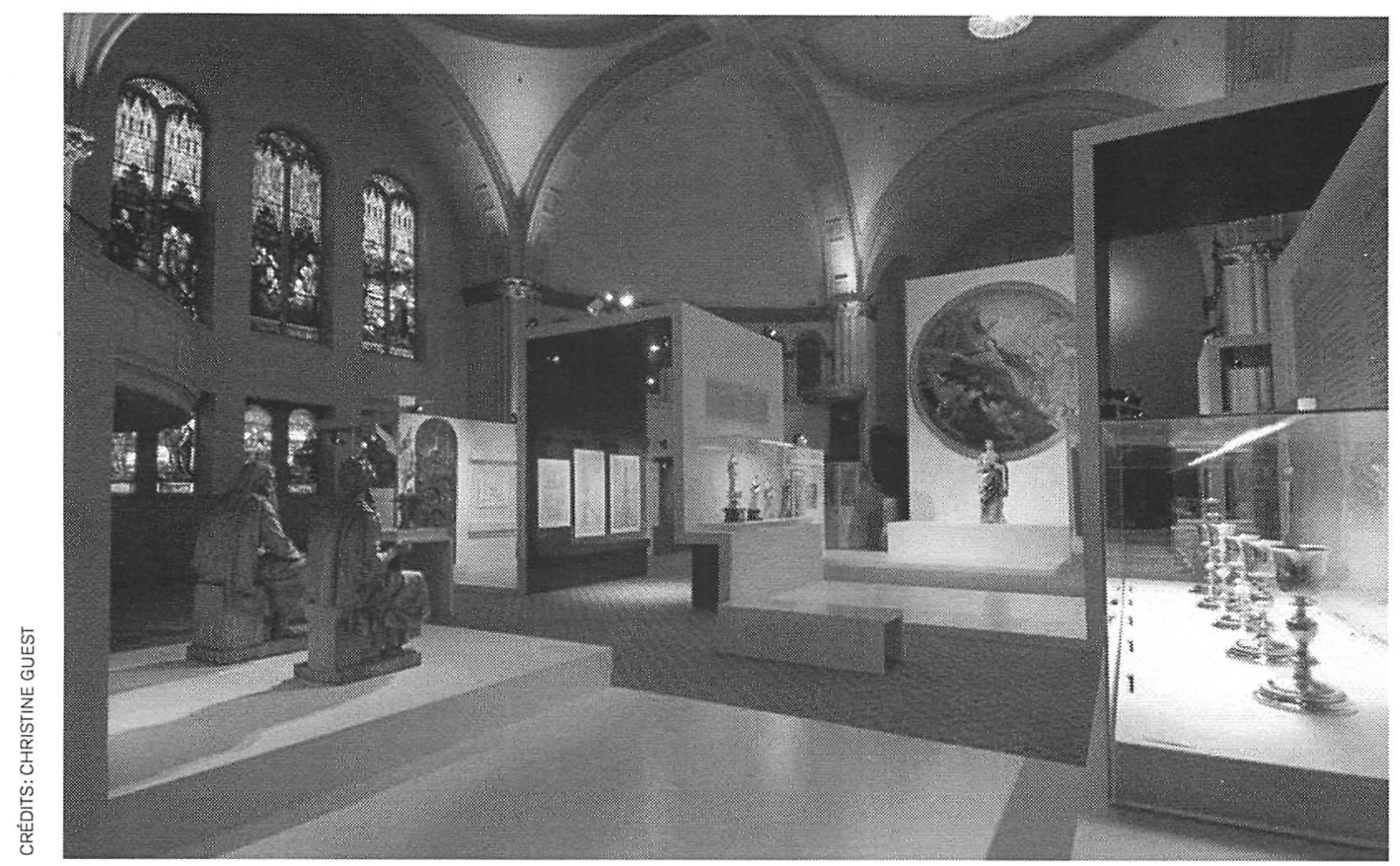

Vue de l'exposition L'Héritage artistique des Sulpiciens de Montréal, dans l'Église Erskine \& American, 2007

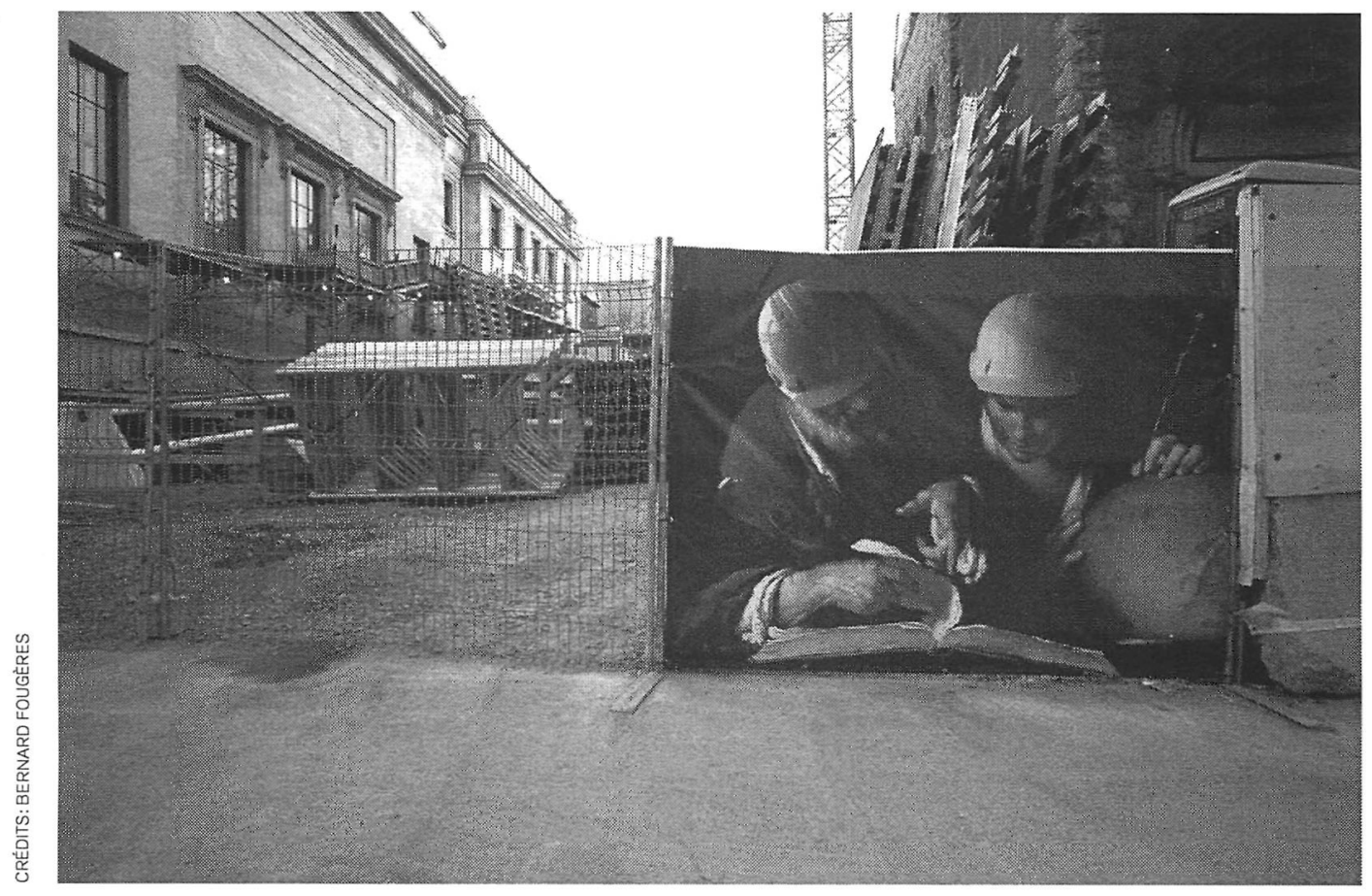

Chantier en cours, nouveau pavillon Claire et Marc Bourgie, nov. 2009 
récemment acquises du musée Marc-Aurèle Fortin avec un design de la firme In Situ.

Au musée, la responsabilité de la mise en exposition inclut le concept d'exposition, la recherche, la selection des œuvres, les contacts avec les prêteurs, l'expographie, la rédaction des textes didactiques et des cartels. La plupart de nos expositions font l'objet d'une publication que nous concevons et coordonnons aussi en collaborant avec divers auteurs et le service des éditions scientifiques du musée. Je dirige présentement la publication d'un premier ouvrage de grande ampleur sur notre collection d'art canadien et québécois, des Premières Nations et des Inuits, qui réunira les textes d'une trentaine d'auteurs.

CATHERINe Fortin \& AlEssandRa MARIANI Quelle est votre ligne directrice pour les nouvelles acquisitions en art canadien?

JACQUes Des Rochers

Il y a d'emblée une part aléatoire dans la constitution des collections de tous les musées, qu'une ligne directrice ait été établie ou non. Nous sommes ensuite à la merci du marché de l'art, des contingences imposées parle temps, et nous devons composer avec la diversité philanthropique des nombreux donateurs. Les ressources monétaires dont nous disposons pour faire l'acquisition d'œuvres ont aussi une très grande incidence. Au-delà de ces considérations de base, il n'y a pas de ligne directrice pertinente sans une connaissance de la collection et de son évolution. Nous décelons alors ses spécificités, ses forces et ses faiblesses, ce qui nous permet d'intervenir adéquatement. Je suis en charge del'art canadien historique qui inclut la production qui s'étend du Régime français au Refus global, de même que de l'art des Premières Nations et de l'art inuit. Bien que mon mandat impliquel'ensemble du Canada, je considère qu'il importe d'abord de favoriser une bonne représentation de Montréal et du Québec, en comblant les lacunes criantes, puis de chercher à représenter le reste $d u$ vaste territoire canadien selon les possibilités. Ainsi,j'ai bien sûr voulu acquérir des œuvres religieuses significatives, absentes des collections. Je pense en particulier à une pièce exceptionnelle de mobilier liturgique du patrimoine montréalais : l'autel du Sacré-Cœur des Sœurs grises de Montréal conçu par Philippe Liébert, daté de 1790. Nous n'avions rien de semblable et Liebert, sculpteur ornementiste remarquable, ne figurait pas au sein de notre collection. En ce qui a trait au XVIII' siècle par exemple, seuls deux portraits anonymes étaient inscrits à la collection; nous avons fait l'achat depuis d'un imposant portrait du premier curé canadien de l'église Notre-Dame de Montréal et un portrait fort rare représentant un architecte franc-maçon, du premier artiste canadien à avoir poursuivi des études dans une académie en France, François Malepart de Beaucourt. 
Dans ce cas, l'œuvre alors inconnue nous a permis de mieux situer le parcours professionnel de l'artiste. Je m'intéresse tout particulièrement au portrait, toutes époques confondues, pour ses qualités psychologiques, au-delà des essentielles valeurs historiques et esthétiques. L'acquisition, par exemple, de l'unique portrait d'Orosco par un Canadien, Stanley Cosgrove, témoigne de l'influence des muralistes mexicains, en plus d'être l'une des œuvres les plus touchantes de cet artiste.

Reconnaître les débuts de la modernité par des œuvres clés ou avec certains artistes méconnus constitue à mon égard une direction qui a une réelle importance. C'est ce qui nous a menés à l'achat d'une Annonciation de Mary Alexandra Bell Eastlake, clairement influencée par les Nabis, et réalisée aussitôt que 1896; il a été aussi possible, par cet achat, de donner place aux femmes. En suivant ce sillon de la modernité, je souhaitais aussi mettre en perspective les débuts de la non-figuration au pays, ce qui s'est concrétisé par l'achat du tableau La vague de Jock Macdonald, daté de 1939, ou encore d'un petit tableau semi figuratif de 1930 de Gordon Webber, ou d'abstractions précoces de Fritz Brandtner.

En ce qui a trait à l'art inuit qui a vu sa mise en marché apparaître à Montréal à la fin des années 1940, l'idée de la constitution d'une telle collection au musée s'est faite très tôt. Il s'agit ici d'une particularité qu'il faut pouvoir conserver. Nous venons, dans cette foulée, de faire l'acquisition d'une pièce d'une rare qualité d'Andy Miki qui date des années 1960. Nous privilégions donc les œuvres anciennes, mais il nous faut cependant combler des lacunes concernant les périodes récentes. Nous avons voulu en particulier mieux représenter les artistes contemporains. Pensons ici à Annie Pootoogook, dont nous avons acheté une œuvre avant qu'elle ne reçoive le prix Sobeys, ou encore à Mattiusi Iyaituk et à Shuvinai Ashoona.

C'est la même chose pour l'art amérindien. Les œuvres majeures de notre collection proviennent essentiellement de la côte nord-ouest $d u$ Canada, alors que peu d'œuvres de notre environnement plus immédiat sont représentées. Puisque les œuvres amérindiennes anciennes se font plutôt rares sur le marché, nous cherchons à les représenter par leur production contemporaine.

CATHERINE FoRTIN \& ALESSANDRA MARIANI Pourquoi avez-vous choisi d'installer la collection d'art canadien dans le nouveau pavillon de l'église Erskine and American United?

\section{JACQUes Des Rochers}

Je réitère le fait que l'idée d'acquérir cette église existait au musée depuis plus de vingt ans, avant même que soit inauguré le pavillon 


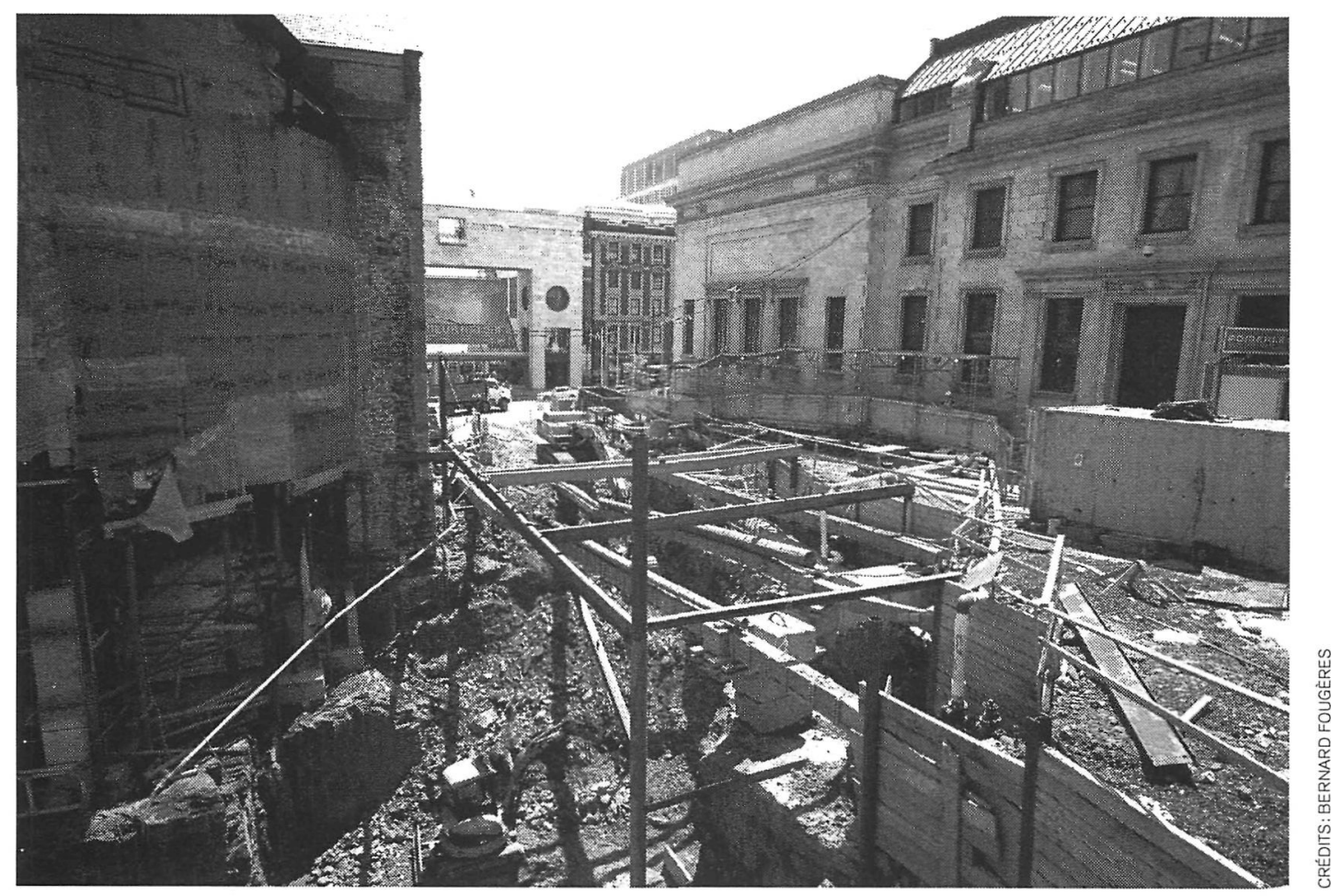

Chantier en cours, nouveau pavillon Claire et Marc Bourgie, nov. 2009 


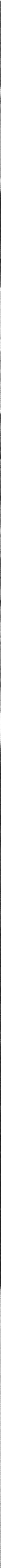


Jean-Noël Desmarais en 1991. Les plans du passage souterrain sous la rue Sherbrooke prévoyaient déjà un lien avec l'église, sousl'avenue du Musée. Ensuite, il a toujours été impensable pour le musée que cette église puisse perdre sa vocation de lieu public, et le processus d'acquisition a été mis en branle dès que son avenir est devenu incertain. Comme vous pouvez le constater, le musée se développe dans un tissu serré et il doit voir où il est possible et avantageux d'étendre ses activités. Au fil du temps, on a proposé pour l'église plusieurs projets, dont une bibliothèque d'art qui aurait regroupé celle du musée et celles des universités montréalaises. Lorsque j'ai été embauché, la disparition progressive des institutions religieuses et la démolition d'églises avaient déjà marqué depuis un bon moment le contexte, mais un projet de préservation et de mise en valeur du patrimoine religieux montréalais était à l'étude pour l'église Erskine and American. La conjonction entre forme et contenu devait en principe aller de soi, mais les œuvres religieuses de qualité étaient difficilement accessibles et c'est pour cette raison que nous avons choisi de mettre en valeur les collections d'art canadien historique, puisque seulement cinq pour cent d'entre elles étaient exposées. Aucune autre collection n'a été considérée pour ce lieu. Entreprendre la restauration de l'intérieur de l'église dans le but de la rendre conforme aux normes muséales de conservation pour nos collections impliquaitle démantèlement des fragiles ornementations de platre et leur entière reconstitution. Le mécénat de la famille Bourgie a toutefois permis d'écarter ce projet hasardeux en suggérant la transformation de la nef en salle de concert. Cette proposition a aussi permis - grâce à la même famille - la création de la Fondation Arte Musica qui veillera au financement et à la programmation musicale. Le musée avait déjà tiré parti avec succès du volet musical qui élargit manifestement le rôle et la portée de l'art dans la société. Cette proposition était une réponse plus appropriée quant àl'utilisation de l'espace contenu dans la nef de l'église : les collections d'art canadien seraient finalement logées dans un nouvel édifice annexé à l'église.

CATHERINe ForTIN \& AlesSANDRA MARIANI Pouvez-vous en dire davantage sur le projet de rénovation de l'église ? Comment ce projet a-t-il été élaboré du point de vue architectural?

JACQUES DES Rochers

Puisqu'il s'agit d'un édifice montréalais important, désignélieu historique national, il allait de soi que la conservation d'un maximum d'eléments significatifs et leur restauration soient assurées. La double fonction envisagée pour les lieux a cependant déterminé la modification de deux sections de l'église. Le chœur qui n'était pas adapté aux besoins à venir sera remplacé par une scène de plus grande dimension, conforme aux standards 
actuels et permettant d'accueillir solistes et orchestres lors de la présentation de programmes musicaux. L'ancienne école du dimanche située à l'arrière de l'église a été démolie pour permettre la construction d'un nouvel édifice de cinq étages qui logera les salles d'exposition. Ces transformations sont justifiées parce qu'elles permettront le maintien d'un lieu de culte qui n'avait pas trouvé de nouvelles fonctions et qui était voué à être banalisé ou à disparâ̂tre. Les nouvelles activités qui investiront les lieux s'apparentent aux activités d'origine: éducation, elévation de l'esprit par les arts et la musique, éducation et contemplation.

À la fin des années 1930, les modifications liturgiques qu'avaient entrainées l'association de deux communautés religieuses différentes, soit l'église Erskine et l'église américaine, avaient elles aussi contraint à une réorganisation des espaces intérieurs, qui avait été réalisée par les architectes Nobbs \& Hyde. L'école et le chœur qui seront remplacés ont été réaménagés à cette époque. Aujourd'hui, comme hier, la forme suit inévitablement la fonction, dans un respect le plus fidèle possible des qualités essentielles du lieu.

Pour présenter de façon un peu plus détaillée le programme architectural, il faut comprendre que notre complexe muséal n'est pas homogène, mais qu'il se compose de plusieurs pavillons d'époques différentes qui ont des structures très diverses. Il a semblé essentiel de donner une certaine cohésion formelle, une certaine "lecture" unificatrice à cet ensemble. Il était également important que le pavillon d'art canadien puisse se démarquer de l'église en revêtant un langage actuel, une facture contemporaine. Le marbre blanc déjà présent dans les deux pavillons principaux du musée est apparu par conséquent comme un choix naturel pour l'enveloppe extérieure du nouveau pavillon, s'inscrivant ainsi dans une intégration séquentielle du développement $d u$ musée et affirmant d'autant plus sa présence. Marbre et verre clair pour la fenestration seront en rupture avec l'église, mais correspondront aux principaux pavillons en maintenant un lien physique et symbolique avec ces derniers, d'autant plus que le marbre sera extrait de la même carrière, au Vermont, d'où ill'a étéil y a plus de cent ans pour le bâtiment d'origine. C'est de cette carrière qu'avait été extrait le fût des colonnes d'un seul bloc pour le pavillon érigé en 1912. De la même façon, les architectes ont tenu à ce que les blocs de marbre choisis pour le nouveau projet témoignent de leur origine et de leur matérialité par une disposition qui donne à lire une tranche intègre et continue de la paroi de la carrière. Ainsi, les quatre façades de la construction nouvelle $d u$ pavillon seront constituées d'une enveloppe de quelque 1500 plaques de marbre. Issues 


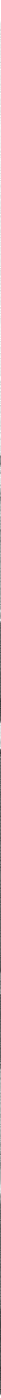

Axonométrie du projet, Provencher Roy + Associés, architectes. 


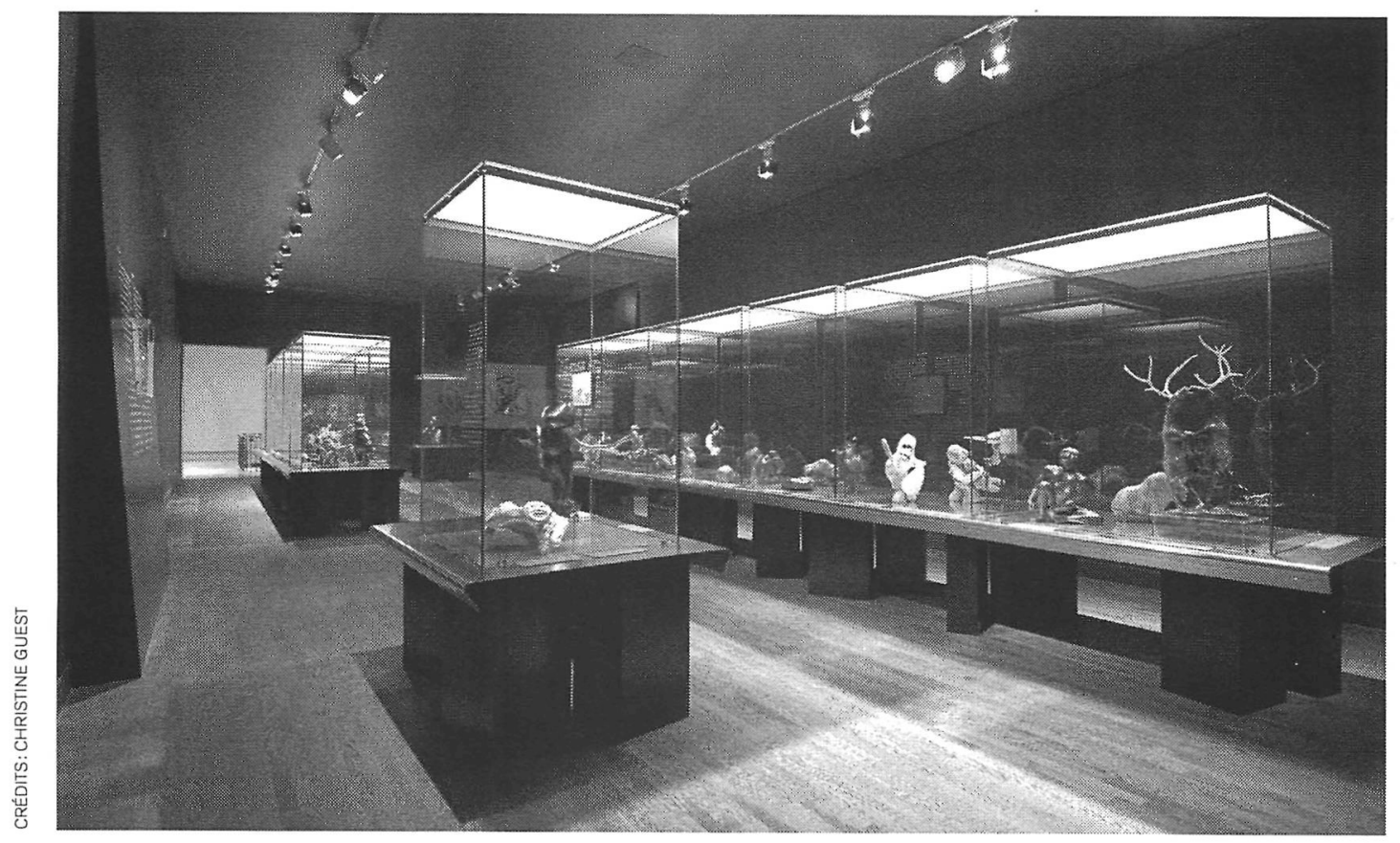

Vue de la collection permanente d'art inuit, Takuminartut, 2006 
d'une même paroi de la carrière, 27 tranches successives de 16 blocs contigus reproduiront sur les façades 27 fois la même configuration de veinures, un appareillage unique en lien avec son contexte.

CATHERINe ForTIN \& ALESSANDRA MARIANI Comment l'architecture et l'esprit du lieu influencerontils la mise en place des expositions? Envisagez-vous une nouvelle façon d'exposer les cuvres?

\section{JACQUES DES ROChERS}

Le volume du pavillon d'art canadien étant déjà différencié dans sa proposition architectonique par rapport à celui de l'église Erskine and American et leurs espaces et fonctions en parties indépendants les uns des autres, il n'a pas semblé pertinent de témoigner du lieu autrement que dans la nef même de l'église restaurée. Comme je l'ai dit précédemment, l'église sera investie par des concerts de musique et divers événements. Le lieu qui contient un ensemble imposant de vitraux, dont une vingtaine provenant de l'Atelier Tiffany, devient, par sa restauration et la conservation de son volume intérieur, la meilleure réponse au maintien de ses qualités identitaires. Je réitère également l'importance de l'adéquation des nouvelles activités avec l'esprit du lieu.

En ce qui a trait au mode d'exposition, le nouvel édifice nous offrira cinq salles de format rectangulaire et de mêmes dimensions qui seront aménagées l'une au-dessus de l'autre afin de donner le plus de latitude possible à la conception de la mise en espace des collections. Une salle d'exposition supplémentaire sera obtenue par le passage souterrain qui relie le nouveau pavillon au pavillon d'origine. Dans notre projet, la mise en exposition générale des collections se veut davantage didactique, en établissant des liens significatifs entre les œuvres et en offrant un support historique à leur compréhension à travers un parcours chronologique, bien que des corpus distincts permettront une approche thématique spécifique, là où c'est justifié. Par ailleurs, chaque étage proposera une mise en espace différente de ces corpus distincts, qu'il s'agisse de la période coloniale ou de l'époque des salons, où les visiteurs pourront découvrir la reconstitution de l'accrochage typique d'un salon, tel celui de l'Art Association of Montreal (ancêtre du Musée des beaux-arts de Montréal), ou qu'il s'agisse des débuts de la modernité ou encore de l'époque des manifestes. Il en sera de même de l'art inuit qui aura aussi son propre étage. Quant à l'apport des Premières Nations, il s'inscrira dans l'ensemble du parcours, appuyé par leurs productions contemporaines ou un regard critique d'autres artistes. 
CATHERINE ForTIN \& AlESSANDRA MARIANI Quelle est l'importance de cet agrandissement dans le positionnement du musée au Québec et sur le plan international?

JACQUES DES ROCHERS

L'intégration d'un lieu de culte dans un complexe muséal d'importance n'est pas une chose encore très commune, bien qu'elle puisse s'imposer pour préserver un certain nombre de ces monuments en déshérence dans les centres historiques. Le musée fera certainement partie d'une courte liste d'exemples pour un certain temps et se démarquera. L'intégration de la musique avec l'envergure que le musée souhaite lui accorder nous positionnera fort probablement parmi les chefs de file de ce genre d'association si je me fiè à ce que mes collègues ont $p u$ observer à l'étranger. Dédier un pavillon entier à la collection d'art canadien et québécois, à l'art des Premières Nations et des Inuits, est en fait une première attendue depuis longtemps.Je suis convaincu que cela donnera un signal fort quant à l'intérêt et à la place que nous voulons accorder à notre identité toute particulière. Notre collection est importante, l'espace qu'on lui allouera en témoignera et permettra à tous, Québécois, Canadiens et étrangers, de mieux la connaître et la reconnaître. 